PSS $\quad \begin{aligned} & \text { PROCEEDINGS } \\ & \text { OF SCIENCE }\end{aligned}$

\title{
On construction methods of the strong gravitation theory
}

\author{
V.M. Koryukin \\ Mari State University \\ 1, Lenin square, Yoshkar-Ola, Russia \\ E-mail:vmkoryukin@gmail.com
}

We propose the quantum theory for the description of gravitation interactions both at large distances and at small ones. This theory can employ both for so named "black holes" and for hadrons.

XXII International Baldin Seminar on High Energy Physics Problems

15-20 September 2014

JINR, Dubna, Russia 
The principle of the theoretical notions adequacy to experimental data must be put in the base of the serious physical theory. It is precisely therefore we attach the fundamental importance to symmetries which's reflect the matter properties in the condensed (pithy) form. For this in the elementary particle physics is used the scattering matrix which allows to guess a form of transition operators if only for linear approximation. Because we must forecast results of future experiments, the description of physical systems states will proceeds by use of smooth functions, which it is desirable to obtain as solutions of differential equations. It is precisely therefore we shall approximate the transition operators by differential operators using the variation formalism. We note that the presence of the Universe neutrino background with the finite Fermi energy $\varepsilon_{F}$ is the catalytic agent of stochastic processes, but the large value of this energy causes to the determinancy of physical processes (we shall use the system of units $h /(2 \pi)$ $=c=1$, where $h$ is the Planck constant and $c$ is the velocity of light). Specifically we connect the large value of the Fermi energy and the low temperature of the Universe neutrino background with the stability (or if only with the metastability) of elementary particles.

In the degenerate state background fermions of Universe, generating Fermi and Bose liquids, are weakly-interacting particles, but it is not excluded by the interaction with hadrons their exhibition as color fermions - ghosts. We do not exclude also the possibility, that in the state of the Fermi liquid they must be considered as right neutrinos and left antineutrinos with the sufficiently high Fermi energy $\varepsilon_{F}$ ("sterile" neutrinos and "sterile" antineutrinos [1]). It must be exhibited in the absence of these particles by decays attributed to weak interactions of low energies (a mirror asymmetry) [1]. Note, that the transition to the classical physics proceeds by use of $\varepsilon_{F} \rightarrow \infty$. We shall consider the covariant gauge theory of strong interactions in the affine connection space, on the base of which may be produced both the quantum chromodynamics and the strong gravitation theory [2]. We regard, that the standard gravitation interaction is not the fundamental one, but it is generated by collective oscillations in the Universe neutrino sea [3]. In consequence of this the weak interaction acquires the global value, because it causes the bond of neutrinos both one with the other and with charged leptons and quarks.

We consider the action which has the form as the following integral

$$
\mathrm{A}=\int_{\Omega_{n}} \Lambda d_{n} V=\int_{\Omega_{n}} \kappa \bar{X}(\Psi) \rho X(\Psi) d_{n} V .
$$

( $\Lambda$ is a Lagrangian). Here and further $\kappa$ is a constant; $\rho=\rho(x)$ is the density matrix ( $\operatorname{tr} \rho=1$, $\rho^{+}=\rho$, the top index "+" is the symbol of the Hermitian conjugation) and the bar means the generalized Dirac conjugation which must coincide with the standard one in particular case that is to be the superposition of Hermitian conjugation and the spatial inversion of the space-time $M_{4}$. We shall name solutions $\Psi(x)$ of differential equations, which are being produced by the requirement of the minimality of the integral (1), as the maximum plausible realizations of Lie local loops $G_{r}(x)$ [2] and we shall use for the construction of the all set of functions $\{\Psi(x)\}$ (generated by the help of the transition operators).

Let $E_{n+N}$ is the vector fiber space with the base $M_{n}$ and the projection $\pi_{N}, \Psi(x)$ is the arbitrary section of fibre bundle $E_{n+N}, \nabla_{i}$ is the covariant derivative symbol. Let us to consider the infinitesimal substitutions defining the vector space mapping of the neighbour points $x$ and $x+\delta x \quad\left(x \in U, \quad x+\delta x \in U, U \subset M_{n}\right)$ and conserving the possible linear 
dependence between vectors. We demand that the action A was the invariant one with respect to the infinitesimal substitutions of the local Lie loop $G_{r}(x)$ conserving the type of geometrical objects. By this the components $C_{a b}{ }^{c}(x)$, alternating on down indices of the structural tensor, must satisfy to the generalized Jacobi identities

$$
C_{[a b}^{d} C_{c] d}^{e}-\xi_{[a}^{i} \nabla_{|i|} C_{b c]}^{e}+\xi_{[a}^{i} \xi_{b}^{j} R_{|i j| c}{ }^{e}=0
$$

$\left(R_{i j c}{ }^{e}(x)\right.$ are the curvature tensor components of the connection $\Gamma_{i a}^{b}(x)$; here and further $x^{i}$ are the co-ordinates of the point $x ; x^{i}+\delta x^{i}$ are the co-ordinates of the point $x+\delta x$; Latin indices $a$, $b, c, d, e$ will run the values of integers from 1 to $\mathrm{r}$; Latin indices $i, j, k, \ldots$ will run the values of integers from 1 to $n$ ).

That the appearance of stringent restrictions can be excluded on a Lagrangian we introduce its dependence on gauge fields $B(x)[2]$. Let

$$
B B^{+}=\rho \operatorname{tr}\left(B B^{+}\right)
$$

in the Lagrangian (1). Further fields $\Psi(x)$ we shall name as prime ones. We denote the components of the gauge fields $B(x)$ as: $B_{a}^{c}(x)$. Probably the rank of the density matrix $\rho$ equals $n$, but it is impossible to eliminate that the given equality is satisfied only approximately when some components of a density matrix can be neglected. In any case we shall consider that among fields $B_{a}^{b}$ the mixtures $\Pi_{a}^{i}$ were formed with non-zero vacuum means $h_{a}^{i}$ which determine differentiable vector fields $\xi_{a}^{i}(x)$ for considered domain $\Omega_{n}$ as:

$$
\Pi_{a}^{i}=B_{a}^{b} \xi_{b}^{i}
$$

(spontaneous breaking of symmetry, fields $\xi_{a}^{i}(x)$ determine a differential of a projection $d \pi$ from $\Omega_{r} \subset M_{r}$ in $\Omega_{n} \subset M_{n}$ ).

Let us rewrite the integral (1) in the following manner

$$
\mathrm{A}_{t}=\int_{\Omega_{n}} \Lambda_{t} d_{n} V=\int_{\Omega_{n}}\left[\Lambda_{\circ}+\Lambda_{1}(\Psi)+\Lambda_{2}(B)\right] d_{n} V,
$$

where $\Lambda_{\circ}$ is the constant which is connected with the normalization and

$$
\begin{gathered}
\Lambda_{1}=\kappa \overline{X^{b}}(\Psi) \rho_{b}^{a} X_{a}(\Psi)=\kappa \bar{D}^{a} \Psi D_{a} \Psi /\left(B_{b}^{\left.+_{b}^{c} B_{c}^{b}\right),}\right. \\
D_{a} \Psi=-B_{a}^{c} X_{c}(\Psi)=B_{a}^{c}\left(\xi_{c}^{i} \nabla_{i} \Psi-L_{c} \Psi\right) .
\end{gathered}
$$

Since the action (5) must be invariant by infinitesimal substitutions of the Lie local loop $G_{r}(x)$, then the Lagrangian $\Lambda_{2}(B)$ must depend on the gauge [1] (boson) fields $B(x)$ by intensities $F_{a b}^{c}(B)$, having the form

$$
F_{a b}^{c}=\Theta_{d}^{c}\left(\Pi_{a}^{i} \nabla_{i} B_{b}^{d}-\Pi_{b}^{i} \nabla_{i} B_{a}^{d}+\Xi_{a b}^{d}\right),
$$

where

$$
\Theta_{b}^{c}=\delta_{b}^{c}-\xi_{b}^{i} \Pi_{i}^{a}\left(B_{a}^{c}-\beta_{a}^{c}\right), \quad \quad \Xi_{a b}^{e}=B_{d}^{e}\left(B_{a}^{c} L_{c b}^{d}-B_{b}^{c} L_{c a}^{d}\right)-B_{a}^{c} B_{b}^{d} C_{c d}^{e} .
$$

Hereinafter a selection of fields $\Pi_{i}^{a}$ and $\beta_{c}^{a}$ are limited by the relations: 


$$
\Pi_{j}^{a} \Pi_{a}^{i}=\delta_{j}^{i}, \quad \quad \beta_{c}^{a} \xi_{a}^{i}=h_{c}^{i} .
$$

Further it is convenient to use the following Lagrangian:

$$
\Lambda_{2}=\frac{\kappa_{\circ}^{\prime}}{4} F_{a b}^{c} F_{g e}^{d}\left[t^{a g}\left(s_{c}^{e} s_{d}^{b}-v s_{c}^{b} s_{d}^{e}\right)+t^{b e}\left(s_{d}^{a} s_{c}^{g}-v s_{c}^{a} s_{d}^{g}\right)+u_{c d}\left(t^{a g} t^{b e}-v t^{a b} t^{g e}\right)\right],
$$

$\left(\kappa_{\circ}^{\prime}, v\right.$ are constants) [1]. If $s_{a}^{b}=\delta_{a}^{b}, t^{a b}=\eta^{a b}, u_{a b}=\eta_{a b}\left(\eta_{a b}\right.$ are metric tensor components of the flat space and $\eta^{a b}$ are tensor components of a converse to basic one) then the given Lagrangian is most suitable one at the description of the symmetry matter (all matter states are equally likely), because it is most symmetrical one concerning intensities of the gauge fields $F_{a b}^{c}$ (within hadrons and within so named "black holes"). What is more we shall require the realization of the correlations:

$$
L_{c d}^{a} \eta^{d b}+L_{c d}^{b} \eta^{d a}=0
$$

that the transition operators $L_{a_{c}}^{b}$ generate the symmetry which follows from the made assumptions.

The transition to the matter description of the observable space region for which one it is possible to suspect that the presence of cluster states of interacting particles will be expressed in following formula for tensors $s_{a}^{b}, t^{a b}, u_{a b}$ and $h_{i}^{a}$ :

$$
\begin{gathered}
s_{a}^{b}=s \xi_{a}^{i} h_{i}^{b}+\xi_{\bar{a}}^{c} \varepsilon_{\underline{c}}^{b}, \quad t^{a b}=t \varepsilon_{(l)}^{a} \varepsilon_{(k)}^{b} \eta^{(l)(k)}+\varepsilon_{\underline{c}}^{a} \varepsilon_{\underline{d}}^{b} \eta^{\underline{c} \underline{d}}, \quad u_{a b}=u \xi_{a}^{i} \xi_{b}^{j} h_{i}^{c} h_{j}^{d} \eta_{c d}+\xi_{\bar{a}} \xi_{b} \underline{d} \eta_{\underline{c} \underline{d}}, \\
h_{i}^{a}=h_{i}^{(k)} \varepsilon_{(k)}^{a} .
\end{gathered}
$$

$((i),(j),(k),(l), \ldots=1,2, \ldots, n ; \underline{a}, \underline{b}, \underline{c}, \underline{d}, \underline{e}=n+1, n+2, \ldots, n+\underline{r} ; \quad \underline{r} / r \ll 1)$, where fields $h_{i}^{(j)}(x)$, taking into account the relations (13), are determined uniquely from equations: $h_{k}^{a} h_{a}^{i}=\delta_{k}^{i}$. Similarly tensors $\eta^{(i)(j)}, \quad \eta^{\underline{a b}}$ are determined from equations: $\eta^{(i)(k)} \eta_{(j)(k)}=\delta_{(j)}^{(i)}$, $\eta^{\underline{a b}} \eta_{\underline{c b}}=\delta_{\underline{\underline{c}}}^{\underline{a}}$, while tensors $\eta_{(i)(j)}, \eta_{\underline{a b}}$ are determined as follows: $\eta_{(i)(k)}=\eta_{a b} \varepsilon_{(i)}^{a} \varepsilon_{(k)}^{b}$, $\eta_{\underline{a b}}=\eta_{c d} \varepsilon_{\underline{a}}^{c} \varepsilon_{\underline{b}}^{d}$. We shall connect constants $\varepsilon_{(i)}^{a}, \varepsilon_{\underline{b}}^{a}$ with a selection of the gauge fields $\Pi_{i}^{a}(x)$ recording them by in the form

$$
\Pi_{i}^{a}=\varepsilon_{(k)}^{a} \Phi_{i}^{(k)}+\varepsilon_{\underline{b}}^{a} \mathrm{P}_{i}^{\underline{b}}
$$

and let $\varepsilon_{\underline{b}}^{a}=0$. Besides we shall apply the decomposition of fields $B_{b}^{a}(x)$ in the form

$$
B_{c}^{a}=\zeta_{i}^{a} \Pi_{c}^{i}+\zeta_{\underline{b}}^{a} A_{\bar{c}}^{\underline{b}},
$$

where $A_{c} \frac{b}{c}=\xi \frac{b}{a} B_{c}^{a}$. Note that we decompose the physical system described by fields $B_{b}^{a}(x)$ on two subsystems. One of them described by fields $\Pi_{a}^{i}(x)$, will play the role of the slow subsystem. In addition components of intermediate tensor fields $\xi_{a}^{i}(x), \xi_{a}^{b}(x), \zeta_{i}^{a}(x), \zeta_{\underline{b}}^{a}(x)$ 
should be connected by the relations: $\zeta_{i}^{a} \xi_{a}^{j}=\delta_{i}^{j}, \zeta_{i}^{a} \xi_{\bar{a}}^{\underline{b}}=0, \zeta_{\underline{b}}^{a} \xi_{a}^{j}=0, \zeta_{\underline{b}}^{a} \xi_{\bar{a}}^{\underline{c}}=\delta_{\underline{b}}^{\underline{c}}$. So, we shall use the reduced set of fields $\left\{\Pi_{c}^{i}(x), A_{c}^{b}(x)\right\}$ instead of the full set $\left\{B_{c}^{a}(x)\right\}$.

Of course, taking into account the absence of a bijection between the real world and the mathematical one, we can construction the maximum plausible physical theory only. It allows using an elemental description, if only for a local domain. We shall use that smooth manifolds are locally diffeomorphic ones to the Euclidean space or to the pseudo-Euclidean space in a certain neighborhood of any point. Therefore we shall choose the connection components $\Gamma_{i}^{b}(x)$ equal to zero in the region under consideration. Since stable states or metastable states are characterized the specific symmetries, then giving the parameter dependence of structural tensor components $C_{a b}^{c}$, we can describe decay processes of elementary particles if only approximately. Specifically, we shall consider that the process of the spontaneous symmetry breaking is characterized the quasi-group structure (we take account of the presence of the Universe neutrino background which is the catalytic agent of stochastic processes, including decays of elementary particles). In consequence of this it is logically connect the stability of differential equations (2) solutions with the stability of elementary particles. As a result functions $C_{a b}^{c}(x)$ must describe the process of spontaneous breaking of symmetry at hadrons decay. Specifically, when $n=r=8$, it allows to do not increase the count of gauge fields beyond 8 as in the grand unified theory. Thereby we consider that gluons are present in the space domain where intermediate vector bosons are absent and on the contrary intermediate vector bosons are present in the space domain where gluons are absent.

Further we shell rely on the cold plasma theory developed for the first-kind superconductor and for the second-kind superconductor. By this a nuclear matter is an analog of second-kind superconductors with respect to gluons, which's as vortices penetrate in a Bose condensate of Cooper pairs compounded from neutrinos, at the same time the vacuum with respect to gluons is an analog of first-kind superconductors. We assume that the interaction energy must depend on a number of particles and quasi-particles participating in this interaction, defining its dependence to space coordinates by means of a mean number of bosons, which's are exchanged two hadrons [4]. As a result ( $n$ is a number of bosons):

$$
E=-2 \int\left\{\sum_{\sigma}\left[\left(\rho_{1} \sigma \rho_{v} \sigma \rho_{2} \sum_{n=0}^{\infty} n e^{-2 n \rho_{v} \sigma r}\right) / \sum_{n=0}^{\infty} e^{-2 n \rho_{v} \sigma r}\right]\right\} d V_{1} d V_{2} .
$$

Because the gravitation theory is constructed for $r \rightarrow 0$ and $r \rightarrow \infty$, then naturally we must state the collision integral (16), retaining only two summands, as

$$
\begin{aligned}
E & =-2 \int\left[\left(\rho_{1} \sigma_{s} \rho_{v} \sigma_{s} \rho_{2} \sum_{n=0}^{\infty} n e^{-2 n \rho_{v} \sigma_{s} r}\right) / \sum_{n=0}^{\infty} e^{-2 n \rho_{v} \sigma_{s} r}\right] d V_{1} d V_{2} \\
& -2 \int\left[\left(\rho_{1} \sigma_{w} \rho_{v} \sigma_{w} \rho_{2} \sum_{n=0}^{\infty} n e^{-2 n \rho_{v} \sigma_{w} r}\right) / \sum_{n=0}^{\infty} e^{-2 n \rho_{v} \sigma_{w} r}\right] d V_{1} d V_{2},
\end{aligned}
$$

where $\rho_{1}$ is the hadron matter density of the first body (a particle), $\rho_{2}$ is the hadron matter 
density of the second body (a particle), $\rho_{v}$ is the density of Universe background neutrinos, $\sigma_{s}$ is the cross-section with the gluon emission or with the gluon absorption (the strong gravitation), $\sigma_{w}$ is the cross-section with the quasi-particle emission or with the quasi-particle absorption in the neutrino collinear beam (spin waves), cementing together two hadrons and produced of the weak interaction (the standard weak gravitation) [3]. Hence discarding the addend in the formula (17), it can obtain [4] the potential as

$$
U(r)=-\frac{C_{S}}{e^{B_{s} r}-1}
$$

( $B_{S} \propto \sigma_{S}, C_{S} \propto \sigma_{S}^{2}$ ) for the description of the strong interaction. Naturally, that at very small distances processes must be described the quantum chromodynamics, but not the strong gravitation theory.

Note that in the more general case, when the connection components $\Gamma_{i_{a}}^{b}(x)$ are not equal to zero and the Lie local loop $G_{r}(x)$ operates in the space of the affine connection as transitively so and effectively, then the correlations (2) become in the Ricci identity ( $C_{a b}^{c}=2 S_{a b}^{c}, S_{a b}^{c}$ are the torsion tensor components of the space $M_{r}$ ). Because the symmetry, characterizing the physical system, is selected in terms of experimental data, the geometrical structure is only the maximum plausible one. Hence it follows that it is desirable to use the spaces of the affine connection with the torsion for the description of particles. What is more precisely the torsion must depend on a rest mass of a particle. The assumption on the "sea" of quarks in the ground state allows using the Landau theory of the Fermi liquid considering observable particles as quasi-particles on the background of "sterile" neutrinos and "sterile" antineutrinos. The properties of the latter's must define the geometrical and topological properties of the spacetime $M_{n}$. We note that the transition to the description of the slow subsystem with the help of the space-time manifold is carried when the Fermi energy $\varepsilon_{F}$ of "sterile" neutrinos tends to infinity. In this case the quotation-marks in the words "sterile" neutrinos can be discarded, because these neutrinos will not collide with the other particles even at very high energies of the latter.

At present veritable elementary observable particles are considered charged leptons. Besides stable leptons are only electrons and positrons. We shell consider them excited states of "sterile" neutrinos and antineutrinos, which's are in the ground state under the temperature $T_{\circ}$ (the temperature of the cosmic microwave background may be its estimation) and the Fermi energy $\varepsilon_{F}$ (the open resonance (126 GeV) at LHC may be its estimation) in the boundary layer of Fermi sphere. The stability condition in the momentum space for the electron is written as

$$
3 \alpha T_{\circ} \varepsilon_{F}^{2} \approx m_{e}^{3}
$$

( $m_{e} \approx 0.5 \cdot 10^{-3} \mathrm{GeV}$ is the electron mass, $\alpha$ is fine structure constant). Here we took into account the Lie algebra dimension of Lie group $S U(2)$, which characterizes the properties of the lepton interior space, and also the equality of the pressure and the temperature for the electron and "sterile" neutrinos. The crude estimation shows $\left(\alpha \sim 10^{-2}, T_{\circ} \sim 10^{-13} \mathrm{GeV}\right.$, 
$\varepsilon_{F} \sim 10^{2} \mathrm{GeV}$ ), that the relationship (19) is satisfied. If we shell consider hadrons, then it need take into account, that the properties of their interior space (in specifically, for the stable proton $(m \approx 1 \mathrm{GeV})$ ) are characterized by group $S U(3)$ (the space dimension $n$ is equal to 8 ). As a result the stability condition (19) in the momentum space for the proton is rewritten in the form:

$$
8 \alpha T_{\circ} \varepsilon_{F}^{7} \approx m_{p}^{8} \text {. }
$$

What is more, considering so named "black holes" as hadrons with the large baryon number [5], we can use the relationship (20) as the stability condition of theirs. By this it is inexpediently the study of the gravitation ignoring the torsion of the space $M_{n}$. Considering formulae (19) and (20) for stable particles we can suggest their generalization for arbitrary particles in the form:

$$
n k T \varepsilon_{F}^{n-1} \approx m^{n},
$$

where the momentum space dimension $n$ of the particle will defined by the number of degrees of freedom. Naturally that the temperature $T$ of the internal space for unstable particles must be over the temperature $T_{0}$.

We suggested considering "black holes" as hadrons with very large baryonic charges [5]. It allows simulating similar objects in laboratory conditions (at high energy accelerators). Naturally, that we connect the use necessity of the quantum chromodynamics in the cosmology with the chance of the processes explanation which's go in quasars and nuclei of Seyfert galaxies with the very large energy release. The Einstein theory cannot apply for this as in it the substantial object - the space-time torsion is absent by the gravitation geometrization (the space-time torsion is the locally diffeomorphic one to the corresponding structural tensor field of the Lie local loop characterizing the symmetry of the quantum system). What is more, in the Hawking process of the "black holes" quantum evaporation is violated the conservation low of the baryonic charge, accumulated the massive collapsing star. If we shall apply this process for the description to two-photon decay of pseudoscalar neutral mesons (this low takes place), then we receive a discrepancy with experimental data (instead the increase of the particles lifetime it is reducing with the growth of their masses).

\section{References}

[1] V.M. Koryukin, The confinement and the dark matter of the Universe, in proceedings of the XIX International Baldin Seminar on High Energy Physics Problems: Relativistic Nuclear Physics and Quantum Chromodynamics, JINR, Dubna 2008, V. 1, XVI, p. 61-66.

[2] V.M. Koryukin, The strong gravitation and the chromodynamics, Sov. J. Nucl. Phys., 1990, Vol. 52, p. 573 - 579.

[3] V. Koryukin, Gravitation and Weak Interactions in the Gauge Fields Covariant Theory, in proceedings of III International Symposium on Weak and Electromagnetic Interactions in Nuclei (JINR, Dubna, Moscow Region, RUSSIA, June 16-22, 1992), Edited by Ts. D. Vylov, Singapore, New Jersey, London, Hongkong: World Scientific, 1992, p. 456 - 458.

[4] V.M. Koryukin, On the quantum theory of fundamental interactions at large distances, Russian Physics Journal, Volume 55, Issue 6 (2012), p. 685-689.

[5] V.M. Koryukin, Two subsystems of the Universe matter and the "black holes", in proceedings of International Scientific Meeting "Physical Interpretations of Relativity Theory - 2013”" (PIRT-2013) (Moscow, BMSTU, 1 - 4 July 2013). Edited by M.C. Duffy, V.O. Gladyshev, A.N. Morozov, P. Rowlands. Moscow: BMSTU, 2013, p. 163-169. 